\title{
Perceived Roles of Fathers in the Promotion, Support and Protection of Breastfeeding
}

Siyabulela E. Mgolozeli

https://orcid.org/0000-0002-1271-0021

University of Pretoria, South Africa

siyabulela.mgolozeli@up.ac.za

\section{Lunic B. Khoza}

https://orcid.org/0000-0003-1368-8759

University of Venda, South Africa

\author{
Hilda N. Shilubane \\ https://orcid.org/0000-0002-6121-0488 \\ University of Venda, South Africa
}

\author{
Cebisa N. Nesamvuni \\ https://orcid.org/0000-0002-4660-2248 \\ University of Venda, South Africa
}

\section{Abstract}

Fathers of young infants have a crucial role to play in the promotion, support and protection of breastfeeding as women often face many challenges during the post-partum period. The World Health Organization recommends paying particular attention to participation by the male partners in child and maternal health as they also have responsibilities and roles to play in nurturing their young ones and supporting their families. The focus of this research was to explore and to describe the perceived roles of fathers in the support, protection and promotion of breastfeeding. This study adopted a qualitative method using an explorative and descriptive design, and the sample included twelve fathers of infants aged less than six months at Schoonoord Village, Sekhukhune District, Limpopo province, South Africa. Using in-depth individual unstructured interviews, data were collected until data saturation was attained and analysed using Tesch's open-coding method. The findings show that fathers perceive breastfeeding to be the optimum feeding choice for the baby and beneficial to the mother. Provision of emotional and financial support to the women, protection of the mother and the baby, motivation and assistance in seeking healthcare emerged as the key roles of fathers during the breastfeeding period. This study recommends that fathers be involved in breastfeeding counselling and that healthcare providers create an environment that is conducive to the participation of fathers in the topic of infant feeding.

Keywords: fathers; roles; promotion; support; protection; breastfeeding

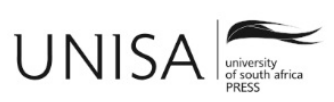




\section{Introduction and Background}

The World Health Organization (WHO 2010, 3) and United Children's Fund (UNICEF 2010) recommends that all babies less than six months be exclusively breastfed and that this continue for up to two years with complementary feeding. Exclusive breastfeeding refers to giving the child only breast milk without feeding any solid food or semi-solid food and drinks, and using medication only as prescribed by a health professional (WHO 2010, 6). Breastfeeding has many health and economic benefits for both the mother and the baby (Heymann, Raub, and Earle 2013, 398). Breastfeeding is reported as the only solution to preventing the outbreak of diarrheal infections that contribute to the high infant mortality rates in sub-Saharan Africa (Victor et al. 2013, 2). It also plays a crucial role in the prevention of post-partum haemorrhage during the postnatal period as an infant feeding from the breast stimulates oxytocin secretion which aids in uterine involution (Cohain 2010, 348).

Furthermore, breastfeeding creates a bond between the mother and the baby (Liu, Leung, and Yang 2013, 76) and is a recognised cost-effective infant feeding option ideal for all babies irrespective of the socio-economic status of the mother (Abdel-Hady, Eladawi, and El-Gilany 2013, 76). With the reported benefits of breastfeeding for both the mother and the baby, the WHO recommends that mothers receive counselling and ongoing support for exclusive breastfeeding at each postnatal contact (WHO 2014, 3). Mothers regard high-quality professional support and guidance as the most important element for successful breastfeeding (Hauck et al. 2011, 55). However, there are other available options such as getting the spouses, family and community involved in supporting and promoting breastfeeding.

Many recently conducted international studies have highlighted the need for the integration of fathers in breastfeeding support and promotion (Chezem 2012, 158; Goosen, McLachlan, and Schübl 2014, 20; Sherriff and Hall 2011, 472). The participation of fathers in breastfeeding is associated with a positive impact on the woman's decision to breastfeed and to maintain breastfeeding (Susin and Giugliani 2008, 386). The role of fathers and male partners in breastfeeding support and promotion is emphasised as crucial to raising breastfeeding rates in developed and developing countries (Brown, Raynor, and Lee 2011, 45). However, fathers often feel unprepared for the challenges breastfeeding can bring (Sherriff and Hall 2011, 472) owing to the fact that they often have feelings of helplessness and exclusion from the breastfeeding process (Chezem 2012, 158; De Montigny and Lacharité 2004, 337).

Given the importance of fathers' involvement in supporting mothers to breastfeed, there is little consideration of fathers' role in supporting and promoting breastfeeding. Research in Western countries suggests that fathers are interested in breastfeeding and want to be involved (Sherriff and Hall 2011, 473) and support the mother throughout the breastfeeding period (Harwood 2011, 8; Stremler and Lovera 2004). Although this has occurred in some regions, most fathers report being poorly informed about the 
benefits of breastfeeding, and identify barriers such as centralising breastfeeding for women only and refusal to allow fathers to attend breastfeeding counselling sessions (Cohen, Lange, and Slusser 2002, 61; Nkuoh et al. 2010). It is reported that fathers in Western cultures do not support mothers breastfeeding in public, feel jealous of the bonding of the mother with the baby and regard the breast as a sexual object (Magnusson et al. 2017, 162).

Frequently men discourage breastfeeding owing to the perception that the breast is for sexual purposes only and force the woman to bottle-feed or formula-feed the baby (Rempel, Rempel, and Moore 2017). Some studies have confirmed that fathers often devalue their role in promoting and supporting breastfeeding as they perceive it to be a woman's issue and her decision (Harwood 2011,33). Culture and community stereotypes are also factors contributing to the lack of fathers' involvement in child and maternal care (Matseke et al. 2017, 16; Nesane, Maputle, and Shilubane 2016, 4). The South African National Strategic Plan for Human Immune Virus, Sexual Transmitted Infections and Tuberculosis calls for the need to strengthen these programmes by engaging fathers (Department of Health 2011). The global steps for successful breastfeeding recommend the involvement of fathers in supporting breastfeeding (WHO 2009; Center for Disease Control Prevention 2011, 1020).

\section{Problem Statement}

Although other programmes such as the prevention of mother-to-child transmission (PMTCT) acknowledge the need for involvement of fathers, the National Infant and Young Child Feeding (NIYCF) Policy does not mention the contribution of fathers in supporting and promoting breastfeeding. This could be an additional factor related to the absence of fathers' involvement in breastfeeding. The rate of breastfeeding in South Africa remains below the target level of 100 per cent coverage (Du Plessis et al. 2016) and the lack of fathers' or family involvement and pressures has been reported as a contributory factor to low breastfeeding rates (Goosen, McLachlan, and Schübl 2014, 26; Jama et al. 2017, 9). There is a paucity of research conducted on the roles of fathers in breastfeeding promotion, support and protection. The few studies that are available have been conducted in developed countries and there is no mention of the involvement of fathers in deep rural areas where cultural stereotypes still exist. Schoonoord Village, which is "deep rural", is not an exception as breastfeeding rates are very low and fathers' involvement in breastfeeding remains a significant challenge owing to cultural stereotypes. The researcher answered the following research question:

- What are the fathers' perceptions of breastfeeding and perceived roles in breastfeeding support, protection and promotion? 


\section{Purpose and Objectives of the Study}

The purpose of this study was to explore and to describe the perceived roles of fathers in the promotion, support and protection of breastfeeding. The objectives of the study were the:

- exploration and description of the perceptions of fathers towards breastfeeding, and

- exploration and description of the perceived roles of fathers regarding breastfeeding support, protection and promotion.

\section{Research Methodology}

\section{Research Design}

A qualitative, explorative, descriptive and contextual research design was used. This helped the researchers to understand the phenomenon from the participants' viewpoint as it materialises (Brink, Van der Walt, and Van Rensburg 2012, 136). It also facilitated data gathering on the perceived roles of fathers in the support, promotion and protection of breastfeeding (Rubin and Babbie 2007, 416). The researcher conducted individual interviews with each participant at a convenient venue and time over a period of five weeks between August and September 2017.

\section{Study Population and Sample}

The study population included all male partners of mothers of infants who were less than six months at Schoonoord Village, Limpopo province. Twelve participants were selected from the study population. A purposive sampling technique was used to select the participants based on the fact that their partners were still breastfeeding their infants. The participants were recruited through their female partners who were attending the breastfeeding support group at Schoonoord Village. The breastfeeding support group was initiated by the first author in 2015 after the local facility where he worked was piloted as the ideal clinic and prepared for accreditation as a mother-baby friendly facility.

\section{Data Collection}

Unstructured individual interviews were conducted to collect data from the 12 participants until data saturation was reached. The main question asked during the interviews was: "Can you explain to me in detail your perceptions about breastfeeding and your role in the support, promotion and protection of breastfeeding?" Further investigation following this question sought clarity and added explanations as participants responded to the central issue. The researcher spent time with the participants and observed all the non-verbal responses that could indicate irritability, stress or discomfort and kept personal notes including his reflections and experience during the interview process (Brink, Van der Walt, and Van Rensburg 2012, 150; Rubin 
and Babbie 2007, 436). Interviews were conducted in Sepedi and transcribed verbatim and later translated to English for analysis.

\section{Data Analysis}

Tesch's open-coding method was used to analyse the data. The interviews were transcribed verbatim and organised into themes and sub-themes. An independent coder verified the results of the open coding and grammar was not changed to avoid losing any information. A literature control was presented after data collection and analysis as it formed a basis for comparing and contrasting the findings of the study (Creswell 2007, 69).

\section{Trustworthiness}

Trustworthiness was maintained throughout this study by using Guba's model (Brink, Van der Walt, and Van Rensburg 2012, 91; Creswell 2009). Credibility was established by the prolonged engagement during which the interviewer spent more time with the participants to establish rapport and to build a relationship based on trust. A voice recorder was also used. Transferability was established through a complete description of the study methodology and the accompanying literature control maintained clarity.

\section{Ethical Considerations}

The Research Ethics Committee of the University of Venda granted ethical approval for this study, and permission to conduct this study was sought from the provincial department of health research committee and the traditional kraal of the village where the data were collected. Verbal informed consent was obtained from all the male partners participating in this study. Privacy and confidentiality were ensured by conducting interviews with the participants in their houses. The researcher explained to the participants that their information would not be shared with anyone and informed them that they would not be forced to answer if they felt that the questions violated their right to confidentiality. The researchers also advised participants that field notes would be taken and a voice recorder used during interviews to capture the proceedings of interview sessions. Participants were assigned codes and their real names were not used to ensure confidentiality and anonymity.

\section{Results and Discussion}

The participants' ages ranged between 19 to 39 years. Four participants were teenage fathers who were not married; eight were adults and were married. All males who participated in this study were fathers of infants less than six months who were breastfed. All participants communicated and expressed themselves well in the Sepedi language. Three main themes emanated from the data analysis, namely perceptions about breastfeeding, perceived roles of fathers in breastfeeding, and perceived hindrances to the roles of fathers in breastfeeding. These are listed in Table 1. 
Table 1: Emerged themes and subthemes

\begin{tabular}{|c|c|c|}
\hline Main theme & Sub-theme & Categories \\
\hline \multirow{4}{*}{$\begin{array}{l}\text { Perceptions of } \\
\text { breastfeeding by fathers of } \\
\text { infants }\end{array}$} & $\begin{array}{l}\text { Breastfeeding as the best } \\
\text { choice for the baby }\end{array}$ & Breast milk is the best \\
\hline & \multirow[t]{3}{*}{$\begin{array}{l}\text { Advantages and benefits of } \\
\text { breastfeeding }\end{array}$} & $\begin{array}{l}\text { Protection of the baby from } \\
\text { illnesses }\end{array}$ \\
\hline & & $\begin{array}{l}\text { Cheap and freely available } \\
\text { at anytime }\end{array}$ \\
\hline & & $\begin{array}{l}\text { Creates bonding and } \\
\text { enhances the mother's love } \\
\text { for the baby }\end{array}$ \\
\hline \multirow{7}{*}{$\begin{array}{l}\text { Perceived roles of fathers in } \\
\text { breastfeeding of infants }\end{array}$} & \multirow[t]{2}{*}{ Supportive role } & Emotional support \\
\hline & & Financial support \\
\hline & \multirow[t]{2}{*}{ Protection } & $\begin{array}{l}\text { Protection against mixed } \\
\text { feeding }\end{array}$ \\
\hline & & $\begin{array}{l}\text { Protection against formula } \\
\text { manufacturers }\end{array}$ \\
\hline & \multirow[t]{3}{*}{$\begin{array}{l}\text { Assistance in seeking } \\
\text { healthcare }\end{array}$} & $\begin{array}{l}\text { Participate in child health } \\
\text { services }\end{array}$ \\
\hline & & $\begin{array}{l}\text { Involvement in PMTCT } \\
\text { programmes }\end{array}$ \\
\hline & & $\begin{array}{l}\text { Seek health information and } \\
\text { alternative healthcare }\end{array}$ \\
\hline \multirow{4}{*}{$\begin{array}{l}\text { Perceived hindrances to the } \\
\text { roles of fathers in } \\
\text { breastfeeding }\end{array}$} & \multirow[t]{2}{*}{ Negative attitudes } & $\begin{array}{l}\text { Negative attitudes from } \\
\text { healthcare workers }\end{array}$ \\
\hline & & Spouse's negative attitudes \\
\hline & \multirow[t]{2}{*}{ Cultural beliefs } & $\begin{array}{l}\text { Breastfeeding concerns } \\
\text { only women }\end{array}$ \\
\hline & & $\begin{array}{l}\text { A man who talks about and } \\
\text { support breastfeeding is not } \\
\text { a real man }\end{array}$ \\
\hline
\end{tabular}

\section{Theme 1: Perceptions about Breastfeeding}

Participants in this study were more positive and confident that breastfeeding is best for all babies of less than six months. They reported that breastfeeding has benefits for both the mother and the baby. Two sub-themes arose during the data analysis under this theme, namely the perception that breastfeeding is the best choice for the baby, and the advantages and benefits of breastfeeding.

\section{Sub-theme 1.1: The Perception that Breastfeeding is the best Choice for the Baby}

The participants in this study stated that breastfeeding is the best choice for the baby and indicated that it was the only option they would recommend for all babies. Furthermore, the participants reported that breastfeeding should be for all babies irrespective of the mother's background, race, socio-economic class and age. This is what the participants said: 
Breast milk is the best and all babies should get the God-given gift. Every woman should give her baby breast milk because it is good for everyone. It does not matter whether you are black, white, Indian, or whether you are rich or poor ... aaah do you get what I mean? Mothers need to know that their breasts are full of life and that the milk they carry is the best! This breast milk cannot be compared with any other meals. Participant 3

I think breastfeeding is the only good option for the baby because it was meant for all the babies. Participant 12

Breast milk is number one. Yes, I chose it for my baby boy and I still think we made the best choice for choosing breast milk for our baby. Every baby is supposed to be given breast milk because it is the best start for a healthy baby. It is the best and you stay away from many troubles. Participant 1

\section{Sub-theme 1.2: Advantages and Benefits of Breastfeeding}

This study found that the participants were aware and knowledgeable about the benefits of breastfeeding. They reported that breastfeeding protects the baby from illnesses, is cheap and freely available all the time and creates a bond between the mother and the baby, which in turn, deepens the woman's love for the baby. This is what participants had to say:

Breastfeeding has many advantages. Aaaah one: it is cheap. No need to buy because it is free from the best. My wife does not have to stress about buying, no need to be stressing about electricity, water and staff. It is easily available at all times. All I can say is that it is free of charge. Participant 8

I have seen with my child that breastfeeding protects the baby from all of these sicknesses. My first baby was not breastfed, we gave him formula milk and he was forever sick. But with this one, we don't have any problem because he is breastfeeding. You know? When the baby is given breast milk, the child does not get sick, no diarrhoea, no coughing. Participant 5

The baby sleeps well when she is given breast milk and I see that she gets used to my wife. I think the more my wife gives her milk is the more this bond develops and love develops all the way. You can see the bond and connection that she has with her mother when she sucks from the breast. It so amazing and it also makes me happy to see the child sleeping and not crying at night. Participant 10

\section{Theme 2: Perceived Roles of Fathers in Breastfeeding of Infants}

\section{Sub-theme 2.1: Supportive Role}

Participants reported that one of their main roles in the promotion of breastfeeding is to provide support to their female partners. Spousal encouragement was a vital issue in facilitating successful breastfeeding among women breastfeeding their infants. There 
are two categories that emerged during analysis under this sub-theme, namely emotional and financial support.

\section{Emotional Support}

The participants indicated that their chief role was to provide emotional support to their female partners who were breastfeeding as it was not always easy. Many participants further argued that females' experience a lot of emotional changes and are sometimes feeling depressed, hence their role is mostly to provide emotional support during difficult times. Two participants had this to say:

My wife experienced a lot of stress and she was forever sad and crying because she did not know whether to continue breastfeeding or not and I made sure that I support her whenever she feels down. Participant 7

My major role is to make sure that my wife is happy and comfortable with everything. I mean how can she breastfeed the baby if she is not happy? I have to make her happy and love her always. Participant 3

Several studies report the need for emotional support for breastfeeding women and that fathers are the first contacts for emotional support and morality (Datta, Graham, and Wellings 2012, 163; Rempel and Rempel 2011, 118; Schmied et al. 2011, 56). A study in Ireland reported that many women avoided and discontinued breastfeeding owing to technical difficulties and inadequate practical and emotional support (Bennett, McCartney, and Kearney 2016, 176). Also a study by Mgolozeli and Shilubane $(2015,92)$ on factors contributing to mixed-feeding practices among mothers and caregivers reported a lack of support from spouses and families as one of the reasons that led to the introduction of solid food stuffs and the discontinuation of breastfeeding.

\section{Financial Support}

The participants also reported that provision of financial support to women was another key role as a lack of financial support to women during breastfeeding can lead to early weaning of the baby as women would want to seek employment to sustain their living. The participants in this study strongly believed that a man is obliged to support his partner financially so that she does not suffer or compromise breastfeeding. One participant further highlighted that women need money to buy food and toiletries from their male partners. This is what participant 4 had to say:

The man is the head of the house, so he should be able to provide financial support to the wife. A wife needs food and cosmetics, so how can she breastfeed well if she is hungry and broke?

This is supported by the findings of a study by Alio et al. $(2013,60)$ that found that the majority of the participants in their study indicated that financial support from male 
partners is important and that they should help maintain the financial bills and provide all the necessities for the house.

\section{Sub-theme 2.2: Protection and Security}

The participants indicated that another important role of male partners in the promotion of breastfeeding is to provide protection and security for the breastfeeding mother. They asserted that it is the responsibility of the father to protect the breastfeeding child from mixed feeding and he is obliged to make sure that the mother does not get tempted to mix-feed the child. They further highlighted that their role is also to protect the women against formula manufacturers who try to convince them to use formula feeding.

\section{Protection against Mixed Feeding}

Protection of the child from mixed feeding and temptation of mothers to mix-feed the child was highlighted as the role of male partners in the promotion of breastfeeding. Male partners were very negative about mixed feeding and they reported that it is their role to protect the child and the mother from such practices. This is what participant 10 had to say:

My major role is to protect my child from mixed feeding because I was told in the clinic that it does not provide good nutrition than the breast. I need to make sure that my wife does not get tempted from such practices.

Participant 8 had this to say:

It is my role as the father to make sure that my wife breastfeeds only and avoid giving other things like porridge and water.

This concurs with Chezem $(2012,157)$ who found that paternal partners who were actively involved in infant feeding demonstrated positive attitudes to breastfeeding and were negative about mixed-feeding practices. A recent study in Limpopo found that the lack of support from spouses and family was reported as a contributing factor to the mixed-feeding temptation (Mgolozeli and Shilubane 2015, 91).

\section{Protection against Formula Manufacturers}

The participants reported that they have a role to protect their female partners from formula manufactures who want to convince and encourage them to use formula feeding. They further argued that formula manufactures undermine breastfeeding, so they have the sole responsibility to protect their female partners from those who would discourage them from breastfeeding. This is supported by the following statements:

I need to make sure that my wife does not get tempted to accept any gift from these formula people because sometimes they just give formula milk for free and our wives would think it's a good thing. Participant 1 
These guys like to visit our homes whenever they hear that there is a small baby and they would come and bribe our wives to stop breastfeeding, so it is my responsibility as the head of the house to protect my wife and baby from those people. Participant 6

As long as it's not breast milk for my child, I will never allow my wife to get in contact of those people, all they think of is to make money and they don't care about the baby. I believe all fathers should try to stop that and protect their families. Participant 12

This is supported by Rempel and Rempel $(2011,118)$ who indicated that one of the roles of fathers in breastfeeding is to defend the mothers from individuals who are advocating for formula manufacturers. Harwood's (2011) study among expectant fathers revealed that they perceived their role as protection against formula milk advisers and manufacturers as fundamental in promoting breastfeeding. They argued that it was their responsibility to discourage their wives from accepting free gifts and samples from formula manufactures.

\section{Sub-theme 2.3: Assistance in Seeking Healthcare}

The participants highlighted that they have a huge role to assist their female partners in seeking healthcare services during breastfeeding. They reported that it is their responsibility as male partners to motivate their female partners to seek help when the need arose during breastfeeding instead of just stopping the breastfeeding. Two subthemes emerged from this theme, namely assistance and participation in child health services, and involvement in PMTCT programmes.

\section{Assistance and Participation in Child Healthcare Services}

The participants highlighted that as fathers of the breastfed infants, they need to assist their female partners in seeking child healthcare services and participate in the process. They reported that as fathers of babies, they believe they have the responsibility of being involved whenever there is a need for child health services and being part of the decision-making. This is what participant 9 had to say:

My major role as the father is to assist my wife to seek healthcare whenever the child is sick, and I need to be there when they make decisions about my child. My role is also to be involved in everything like immunisations and I should be there when they introduce other food stuffs as well.

This concurs with Bennett, McCartney, and Kearney $(2016,174)$ who found that the majority of fathers in their study reported a high need for their involvement during the delivery of child healthcare services. However, this differs from Nesane, Maputle, and Shilubane (2016, 3-4) who reported that some male partners who participated in their study were not willing to participate in maternal healthcare as they perceived it to be a women's issue and that their culture contributed to that perception. 


\section{Getting Involved in PMTCT Programmes}

The participants further highlighted that their role is to be involved in HIV counselling and testing for both the mother and the baby. They demonstrated good insight into the EMTCT programmes and they reported that since they loved their children and wives, they did not see any reason why they should be left out. This is what participant 5 had to say:

My role as the father of the child is to help my wife and get involved in the HIV programme that will assist to avoid the transmission of HIV to the child. We have five years now living with HIV and I understand it very well, so when she goes for treatment, I need to be there as the father so that we get the same breastfeeding information together.

This differs from Kidero's $(2014,34)$ study among male partners in Machakos, Kenya, where they found that many male partners were reluctant to participate in EMTCT programmes as they believed that payment for such services was their only role. Many studies revealed that male partners had negative attitudes to EMTCT programmes and this led to a failure to disclose the HIV status of their spouses (Morfaw et al. 2013, 7).

The results demonstrate that the participants are aware of what should happen in protecting and promoting breastfeeding. These findings are contrary to the study results of Mithani et al. $(2015,253)$ which indicated that fathers are keen to support breastfeeding but lack knowledge of what is expected of them. Alternatively, this study could agree with Cohen, Lange, and Slusser $(2002,64)$ who stated that fathers can be enabled to support their partners when they are equipped with breastfeeding knowledge.

\section{Theme 3: Perceived Hindrances to Fulfilling the Roles of Fathers in Breastfeeding}

It came to light during discussions with the participants that even though they were positive about breastfeeding, they also experienced some hindrances. Two sub-themes emanated from this theme, namely negative attitudes and cultural beliefs were hindrances to their role as fathers in breastfeeding. Negative attitudes from healthcare workers and negative attitudes from their spouses hindered their role in protection, support and promotion of breastfeeding. Cultural beliefs such as the belief that breastfeeding is a woman's issue and discrimination of a man who supports and promote breastfeeding were also reported as factors that discouraged men to participate in breastfeeding.

\section{Sub-theme 3.1: Negative Attitudes}

Participants reported that they experiences negative attitudes from the healthcare workers and their spouses. These were reported as barriers that discouraged fathers from participating in breastfeeding and also discouraged them in supporting their partners who were breastfeeding. The participants revealed that healthcare workers in clinics and 
hospitals do not accommodate them when they counsel women on infant feeding and that they felt ignored and neglected. Furthermore, some participants indicated that nurses in the clinics chase them away whenever they accompany their spouses to seek healthcare and reported that some nurses would question their presence or treat them badly. This is what participants said:

I like driving my family to the clinic, but the nurses there treat us somehow ... they are not kind to us at all. You cannot enter their consultation rooms with your wife freely, instead many questions will be thrown on you. Participant 12

I do not like the attitude of nurses and doctors in the hospital because it makes one uncomfortable. When they teach our partners about breastfeeding, they do not involve us and I find it to be unfair. Participant 7

I remember one sister was teaching all women in the clinic about breastfeeding and its benefits. She did not appreciate my presence, instead she requested me to go out because she was teaching about women issues ... yes, that is what she said. Participant 1

The participants also reported that they experienced negative attitudes from their spouses or partners regarding their role in the protection, support and promotion of breastfeeding. They were sometimes seen as too controlling or too much concerned about domestic issues and some participants reported that their female partners were reluctant in allowing them to participate in HIV, infant feeding or breastfeeding counselling. This is supported by the following statements:

As much as I like helping and supporting my wife on breastfeeding and sometimes take her to the clinic. In most cases she becomes very negative about my role and she will always discourage me and tell me that she will be fine and that I shouldn't be concerned when she gives other foodstuffs as she gets advices from nurses. Participant 5

My wife does not like it when I ask if she has breastfed the baby or not. The only answer I will get from her is that she knows what's good for her baby and that I shouldn't be too concerned about kitchen issues because the way she feeds the baby is her own responsibility. Participant 10

My girlfriend gives me a bad attitude whenever I mention the benefits of breastfeeding to her. I remember one day she said to me I want her to get thin because this baby is sucking her breasts 24 hours and this is making her lose weight. She shut me off and said I shouldn't be interfering because it is a woman's choice to breastfeed. Participant 11

It is reported in many studies that men are often reluctant to participate in child and maternal health issues (Dumbaugh et al. 2014, 269; Mitchell-Box and Braun 2013, 476; Nesane, Maputle, and Shilubane 2016, 4). However, this study found that fathers were keen to support, protect and promote breastfeeding although they were often discouraged by negative attitudes from healthcare worker and their female partners. A 
study conducted by Ladur, Colvin and Stinson (2015, 6201) in Cape Town reported that men were hesitant to participate in health services owing to the stigma and negative attitudes from nurses. It is also reported that the lack of partner communication, mistrust and belief in patriarchy are also some of the reasons why men do not participate in PMTCT activities and that women often exclude their male partners (Morfaw et al. 2013, 7).

\section{Sub-theme 3.2: Cultural Beliefs}

Cultural beliefs were reported by fathers as a hindrance to their participation in the promotion, support and protection of breastfeeding. The participants reported that breastfeeding was often regarded as a women's issue and that a man who talks and supports breastfeeding was not regarded as a real man. This is supported by the following statements:

It's difficult for a man to really participate and support his wife on breastfeeding because our culture does not support that. You know the way we were raised and groomed up as men was that as a man you only focus on men's issues not to mingle in women's issues. The feeding of a child is a responsibility of the mother. Participant 9

In our culture, there is a belief that things like breastfeeding are only for females. Participant 10

Yes, our culture does not allow men to be involved in issues of breastfeeding or how the woman gives birth. Yah, but because I understand these things better now, I support my wife irrespective of those cultural beliefs because at the end the baby is mine and I need to make sure that this woman is doing what is right for our child. Participant 4

A man who is more concerned about these things is usually laughed at and not regarded as a real man in our culture. The community expects a man to be out there looking for cows, drinking beer and doing heavy job, not house chores. Breastfeeding is mostly regarded as women's job and that if you are a man you don't associate yourself with those. Participant 2

In our culture they will just say you not man enough. Participant 8

Caring fathers who support their families in breastfeeding are described as superheroes or sexy exotic men, on the other hand, they are perceived as weak or useless (Höfner, Schadler, and Richter 2011, 685). These findings concur with a study conducted in Uganda on the role of male partners in maternal health where men believed that issues related to pregnancy and childbirth were the domain of women and their participation was confined strictly to traditional gender roles (Singh, Lample, and Earnest 2014, 6). Similarly, a study conducted in Cameroon found that male partner participation in child and maternal health issues is affected by sociocultural casts that are associated with tribal beliefs and traditional gender roles (Nkuoh et al. 2010, 367). 


\section{Limitation}

This study focused on fathers of infants less than six months old from one village in the Limpopo province. Their perceived roles in the promotion, support and protection of breastfeeding may be different from those in other regions, therefore, the findings of this study cannot be generalised to other groups.

\section{Recommendations}

This study recommends that male partner involvement in the promotion of infant and young child feeding be strengthened. All health facilities providing child and maternal healthcare should create an environment that is conducive to the involvement of fathers in breastfeeding. An open community-centred approach for breastfeeding should be initiated so that all the relevant stakeholders can take part. The importance of participation by fathers in breastfeeding should be emphasised throughout the pregnancy, labour and during the early days of infant life, and women should be encouraged to invite their male partners to participate in breastfeeding counselling sessions. In practice, healthcare workers should create an environment that is conducive to the participation of fathers in breastfeeding counselling sessions. This study further calls for the inclusion of fathers or male partners in child and maternal health issues, and that the male-partner involvement be included in the NIYCF Policy.

\section{Conclusion}

This study investigated the perceived roles of fathers in the promotion, protection and support of breastfeeding at the Schoonoord Village in the Limpopo province. The main question that guided the study was: "What are the fathers' perceptions of breastfeeding and perceived roles in breastfeeding support, protection and promotion?” Fathers who participated in this study had positive attitudes to breastfeeding and they reported that breastfeeding is the best feeding choice for their babies. The participants demonstrated knowledge of the benefits of breastfeeding such as protection of the baby from illnesses and that breastfeeding is cost-effective (cheap). These are new insights that this study brings to the existing body of knowledge as many studies report a lack of knowledge and negative attitudes of fathers as some of the factors that lead to cessation of breastfeeding. In some studies it is reported that men felt jealous when the baby sucks on the breast and that some believed that breasts were for sex purposes only. Different roles in support, protection and promotion of breastfeeding were reported by the participants in this study and these include: support; protection and security; and assistance in seeking healthcare. These were positives that distinguish this study from other studies as men in other studies were reluctant to participate in child maternal health issues owing to the belief that issues like breastfeeding are predominantly female issues. Few studies have been conducted on the support needs of breastfeeding women and one of the key issues is the lack of support from their male partners. 
This study brought to light that men are willing to support their partners on breastfeeding both emotionally and financially. Fathers reported that their role in breastfeeding is to protect their babies against mixed-feeding practices and to protect the mothers from formula manufacturers. It is well documented in literature that men are reluctant to participate in healthcare services with their partners. However, this study found that fathers perceived their role to also assist their female partners in seeking healthcare. Their role of seeking healthcare includes participation in child healthcare services and getting involved in PMTCT programmes. Although fathers in this study were more positive about breastfeeding and understood their roles, they also experienced some hindrances in playing their role to support, protect and promote breastfeeding. Fathers reported negative attitudes from healthcare workers and their female partners as factors that were barriers to their participation in breastfeeding. Cultural beliefs were also reported as one of the hindrances experienced by fathers in promotion and support of breastfeeding. This study adds value to the existing body of knowledge because men are well known as people who are reluctant to play a role in breastfeeding promotion. This paper provides baseline data that men are not negative as literature states and that the best solution to combat childhood illnesses associated with lack of breastfeeding is to involve male partners in the promotion, support and protection of breastfeeding.

\section{Acknowledgements}

Fathers of all infants less than six months who were on exclusive breastfeeding are greatly appreciated for sharing their perceptions and roles in the support, protection and promotion of breastfeeding. The authors would like to thank the mothers as well for convincing their partners to participate in the study.

\section{Authors' Contributions}

S.E.M. was involved in the conceptualisation, data collection, analysis, literature search and writing of the manuscript. H.N.S. conceptualised, analysed the data and wrote the manuscript. L.B.K was involved in the conceptualisation, data analysis and writing of the manuscript. C.N.N was involved in the data analysis, literature review and manuscript writing.

\section{References}

Abdel-Hady, Doaa, Noha Eladawi, and Abdel-Hady El-Gilany. 2013. "Knowledge of Female Medical Students about Breastfeeding.” Universal Journal of Public Health 1 (3): $72-78$. https://doi.org/10.13189/ujph.2013.010305.

Alio, Amina P., Cindi A. Lewis, Kenneth Scarborough, Kenn Harris, and Kevin Fiscella. 2013. "A Community Perspective on the Role of Fathers during Pregnancy: A Qualitative Study.” BMC Pregnancy and Childbirth 13 (1): 60. https://doi.org/10.1186/1471-2393-1360. 
Bennett, Annemarie E., Daniel McCartney, and John M. Kearney. 2016. "Views of Fathers in Ireland on the Experience and Challenges of Having a Breast-Feeding Partner.” Midwifery 40:169-176. https://doi.org/10.1016/j.midw.2016.07.004.

Brink, Hilla, Christa Van der Walt, and Gisela van Rensburg. 2012. Fundamental of Research Methodology for Health Care Professionals. 3rd ed. Cape Town: Juta.

Brown, A. E., P. Raynor, and M. D. Lee. 2011. "Young Mothers who Choose to Breast Feed: The Importance of being Part of a Supportive Breast-Feeding Community.” Midwifery 27 (1): 53-59. https://doi.org/10.1016/j.midw.2009.09.004.

Center for Disease Control Prevention. 2011. "Vital Signs: Hospital Practices to Support Breastfeeding - United States, 2007 and 2009.” Morbidity and Mortality Weekly Report (MMWR) 60 (30): 1020-25.

Chezem, Jo Carol. 2012. "Breastfeeding Attitudes among Couples Planning Exclusive Breastfeeding or Mixed Feeding.” Breastfeeding Medicine 7 (3): 155-62. https://doi.org/10.1089/bfm.2011.0024.

Cohain, Judy Slome. 2010. "Towards a Physiological Management of the Third Stage that Prevents Postpartum Haemorrhage.” Midwifery Digest 20 (3): 348-51.

Cohen, Rona, Linda Lange, and Wendy Slusser. 2002. “A Description of a Male-Focused Breastfeeding Promotion Corporate Lactation Program.” Journal of Human Lactation 18 (1): 61-65. https://doi.org/10.1177/089033440201800111.

Creswell, John W. 2007. “Five Qualitative Approaches to Inquiry.” Qualitative Inquiry and Research Design: Choosing among Five Approaches, 53-80. 2nd ed. Thousand Oaks: Sage.

Creswell, John W. 2009. Research Design: Qualitative, Quantitative, and Mixed Methods Approaches. 3rd ed. Thousand Oaks: Sage.

Datta, Jessica, Berni Graham, and Kaye Wellings. 2012. “The Role of Fathers in Breastfeeding: Decision-Making and Support.” British Journal of Midwifery 20 (3): 15967. https://doi.org/10.12968/bjom.2012.20.3.159.

De Montigny, Francine, and Carl Lacharité. 2004. "Fathers’ Perceptions of the Immediate Postpartal Period.” Journal of Obstetric, Gynecologic and Neonatal Nursing 33 (3): 32839. https://doi.org/10.1177/0884217504266012.

Department of Health. 2011. South Africa's National Strategic Plan for a Campaign on Accelerated Reduction of Maternal and Child Mortality in Africa (CARMMA). Pretoria: Department of Health Publications. 
Dumbaugh, Mari, Charlotte Tawiah-Agyemang, Alexander Manu, Guus H. A. ten Asbroek, Betty Kirkwood, and Zelee Hill. 2014. "Perceptions of, Attitudes towards and Barriers to Male Involvement in Newborn Care in Rural Ghana, West Africa: A Qualitative Analysis.” BMC Pregnancy and Childbirth 14 (1): 269. https://doi.org/10.1186/14712393-14-269.

Du Plessis, Lisanne, Nazia Peer, Rene English, and Simone Honikman. 2016. "Breastfeeding in South Africa: Are We Making Progress?” South African Health Review 2016 (1): 109_ 23. https://hdl.handle.net/10520/EJC189314.

Goosen, C., M. H. McLachlan, and C. Schübl. 2014. "Factors Impeding Exclusive Breastfeeding in a Low-Income Area of the Western Cape Province of South Africa." Africa Journal of Nursing and Midwifery 16 (1): 13-31. https://doi.org/10.25159/25205293/1484.

Harwood, Katrina. 2011. "Intent of Expecting Fathers to Encourage Breastfeeding, Perceptions of Support and Barriers to Encouraging Breastfeeding.” Master’s thesis, University of Nebraska-Lincoln.

Hauck, Yvonne L., Jennifer Fenwick, Satvinder S. Dhaliwal, Janice Butt, and Virginia Schmied. 2011. “The Association between Women’s Perceptions of Professional Support and Problems Experienced on Breastfeeding Cessation: A Western Australian Study.” Journal of Human Lactation 27 (1): 49-57. https://doi.org/10.1177/0890334410386956.

Heymann, Jody, Amy Raub, and Alison Earle. 2013. "Breastfeeding Policy: A Globally Comparative Analysis.” Bulletin of the World Health Organization 91 (6): 398-406. https://doi.org/10.2471/BLT.12.109363.

Höfner, Claudia, Cornelia Schadler, and Rudolf Richter. 2011. "When Men Become Fathers: Men's Identity at the Transition to Parenthood.” Journal of Comparative Family Studies 42 (5): 669-86. https://www.jstor.org/stable/41604478.

Jama, Ngcwalisa Amanda, Aurene Wilford, Zandile Masango, Lyn Haskins, Anna Coutsoudis, Lenore Spies, and Christiane Horwood. 2017. "Enablers and Barriers to Success among Mothers Planning to Exclusively Breastfeed for Six Months: A Qualitative Prospective Cohort Study in KwaZulu-Natal, South Africa.” International Breastfeeding Journal 12 (43): 1-13. https://doi.org/10.1186/s13006-017-0135-8.

Kidero, Eunice Aura Ongweny. 2014. "Exploring Male Attitudes on Involvement in Antenatal Care: The Case of Prevention of Mother-to-Child Transmission of HIV in Athi River SubLocation of Mavoko Constituency, Machakos County.” Master's thesis, University of Nairobi. http://erepository.uonbi.ac.ke/handle/11295/73607.

Ladur, Alice Norah, Christopher J. Colvin, and Kathryn Stinson. 2015. "Perceptions of Community Members and Healthcare Workers on Male Involvement in Prevention of Mother-to-Child Transmission Services in Khayelitsha, Cape Town, South Africa.” PLoS One 10 (7): e0133239. https://doi.org/10.1371/journal.pone.0133239. 
Liu, Jianghong, Patrick Leung, and Amy Yang. 2013. "Breastfeeding and Active Bonding Protects against Children’s Internalizing Behavior Problems.” Nutrients 6 (1): 76-89. https://doi.org/10.3390/nu6010076.

Magnusson, Brianna M., Callie R. Thackeray, Sarah A. van Wagenen, Siena F. Davis, Rickelle Richards, and Ray M. Merrill. 2017. "Perceptions of Public Breastfeeding Images and their Association with Breastfeeding Knowledge and Attitudes among an Internet Panel of Men Ages 21-44 in the United States.” Journal of Human Lactation 33 (1): 15764. https://doi.org/10.1177/0890334416682002.

Matseke, Motlagabo G., Robert A. C. Ruiter, Violeta J. Rodriguez, Nicole A. Barylski, Stephen M. Weiss, Deborah L. Jones, Karl Peltzer, Geoffrey Setswe, and Sibusiso Sifunda. 2017. “A Qualitative Exploration of the Meaning and Understanding of Male Partner Involvement in Pregnancy-Related Care among Men in Rural South Africa.” Journal of Social, Behavioral and Health Sciences 11 (1): 16.

Mgolozeli, S. E., and H. N. Shilubane. 2015. “Factors Contributing to Mixed Feeding Practices amongst Mothers and Caregivers of Neonates at Maloma Village, Limpopo Province, South Africa.” African Journal for Physical Health Education, Recreation and Dance Supplement:84-94.

Mitchell-Box, Kristen M., and Kathryn L. Braun. 2013. “Impact of Male-Partner-Focused Interventions on Breastfeeding Initiation, Exclusivity, and Continuation.” Journal of Human Lactation 29 (4): 473-9. https://doi.org/10.1177/0890334413491833.

Mithani, Yasmin, Zahra Shaheen Premani, Zohra Kurji, and Shehnaz Rashid. 2015. "Exploring Fathers' Role in Breastfeeding Practices in the Urban and Semiurban Settings of Karachi, Pakistan.” Journal of Perinatal Education 24 (4). https://doi.org/10.1891/10581243.24.4.249.

Morfaw, Frederick, Lawrence Mbuagbaw, Lehana Thabane, Clarissa Rodrigues, Ana-Paula Wunderlich, Philip Nana, and John Kunda. 2013. "Male Involvement in Prevention Programs of Mother to Child Transmission of HIV: A Systematic Review to Identify Barriers and Facilitators.” Systematic Reviews 2 (5):1-13. https://doi.org/10.1186/2046-4053-2-5.

Nesane, Kenneth, Sonto M. Maputle, and Hilda Shilubane. 2016. “Male Partners’ Views of Involvement in Maternal Healthcare Services at Makhado Municipality Clinics, Limpopo Province, South Africa.” African Journal of Primary Health Care and Family Medicine 8 (2): 1-5. https://doi.org/10.4102/phcfm.v8i2.929.

Nkuoh, Godlove N., Dorothy J. Meyer, Pius M. Tih, and Joseph Nkfusai. 2010. "Barriers to Men's Participation in Antenatal and Prevention of Mother-to-Child HIV Transmission Care in Cameroon, Africa.” Journal of Midwifery and Women's Health 55 (4): 363-9. https://doi.org/10.1016/j.jmwh.2010.02.009. 
Rempel, Lynn A., and John K. Rempel. 2011. "The Breastfeeding Team: The Role of Involved Fathers in the Breastfeeding Family.” Journal of Human Lactation 27 (2): 115-21. https://doi.org/10.1177/0890334410390045.

Rempel, Lynn A., John K. Rempel, and Katrina C. J. Moore. 2017. "Relationships between Types of Father Breastfeeding Support and Breastfeeding Outcomes.” Maternal and Child Nutrition 13 (3). https://doi.org/10.1111/mcn.12337.

Rubin, Allen, and Earl Babbie. 2007. Research Methods for Social Work. 6th ed. Belmont: Cengage Learning.

Schmied, Virginia, Sarah Beake, Athena Sheehan, Christine McCourt, and Fiona Dykes. 2011. "Women's Perceptions and Experiences of Breastfeeding Support: A Metasynthesis." Birth 38 (1): 49-60. https://doi.org/10.1111/j.1523-536X.2010.00446.x.

Sherriff, Nigel, and Valerie Hall. 2011. "Engaging and Supporting Fathers to Promote Breastfeeding: A New Role for Health Visitors?” Scandinavian Journal of Caring Sciences 25 (3): 467-75. https://doi.org/10.1111/j.1471-6712.2010.00850.x.

Singh, Debra, May Lample, and Jaya Earnest. 2014. “The Involvement of Men in Maternal Health Care: Cross-Sectional, Pilot Case Studies from Maligita and Kibibi, Uganda.” Reproductive Health 11 (68): 1-8. https://doi.org/10.1186/1742-4755-11-68.

Stremler, Jewell, and Dalia Lovera. 2004. "Insight from a Breastfeeding Peer Support Pilot Program for Husbands and Fathers of Texas WIC Participants.” Journal of Human Lactation 20 (4): 417-22. https://doi.org/10.1177/0890334404267182.

Susin, Lulie Rosane Odeh, and Elsa Regina Justo Giugliani. 2008. "Inclusion of Fathers in an Intervention to Promote Breastfeeding: Impact on Breastfeeding Rates.” Journal of Human Lactation 24 (4): 386-92. https://doi.org/10.1177\%2F0890334408323545.

UNICEF (United Children’s Fund). 2010. “Breastfeeding.” Accessed 27 September 2018. https://www.unicef.org/breastfeeding/.

Victor, Rose, Surinder K. Baines, Kingsley E. Agho, and Michael J. Dibley. 2013. "Determinants of Breastfeeding Indicators among Children less than 24 Months of Age in Tanzania: A Secondary Analysis of the 2010 Tanzania Demographic and Health Survey.” BMJ Open 3 (1): e001529. https://doi.org/10.1136/bmjopen-2012-001529.

WHO (World Health Organization). 2009. Infant and Young Child Feeding: Model Chapter for Textbooks for Medical Students and Allied Health Professionals. Geneva: WHO.

WHO (World Health Organization). 2010. Indicators for Assessing Infant and Young Child Feeding Practices Part 3: Country Profiles. Geneva: WHO.

WHO (World Health Organization). 2014. WHO Recommendations on Postnatal Care of the Mother and Newborn. Geneva: WHO. 